\title{
EFEITOS DA IDADE E CLASSE DIAMÉTRICA NA DEFORMAÇÃO RESIDUAL LONGITUDINAL EM ÁRVORES DE Eucalyptus dunnii Maiden ${ }^{1}$
}

\author{
Paulo Fernando Trugilho², Setsuo Iwakiri³ ${ }^{3}$ Márcio Pereira da Rocha ${ }^{3}$, Jorge Luiz Monteiro de Matos ${ }^{3}$, \\ Leopoldo Karman Saldanha ${ }^{4}$
}

\begin{abstract}
RESUMO - O objetivo do trabalho foi avaliar os efeitos da idade e da classe diamétrica nos níveis das tensões de crescimento longitudinal em árvores de Eucalyptus dunnii oriundas de plantio comercial. O material avaliado foi procedente da Empresa Procopiak Compensados e Embalagens S.A., localizada no município de Canoinhas, Santa Catarina. Os níveis de tensão de crescimento foram mensurados indiretamente pelo método do "CIRADForêt". Os resultados indicaram que os níveis da deformação residual longitudinal (DRL) apresentaram tendência de aumento linear com a idade. O efeito da classe diamétrica evidenciou correlação negativa com a DRL aos 8 e 13 anos de idade.
\end{abstract}

Palavras-chave: Eucalyptus dunnii, madeira, idade da árvore, tensões de crescimento.

\section{AGE AND DIAMETRIC CLASS EFFECTS ON LONGITUDINAL RESIDUAL STRAIN IN Eucalyptus dunnii TREES}

\begin{abstract}
This research aims at evaluating the age and diametric class effects on levels of longitudinal residual strain in trees of Eucalyptus dunnii originated from commercial plantation. The appraised material came from Procopiak Company-Plywood and Package, located in Canoinhas, State of Santa Catarina-Brazil. The growth stress levels were measured indirectly through the "CIRAD-Forêt" method. The results indicated that the levels of longitudinal residual strain (DRL) showed a tendency for linear increase with age. The diametric class effect gave negative correlation with DRL for 8 and 13-year old trees.
\end{abstract}

Key words: Eucalyptus dunnii, wood, tree age, growth stress.

\section{INTRODUÇÃO}

De acordo com Assis (1999), os atributos que tornam importante o gênero Eucalyptus como fonte de matéria-prima fabril são a sua capacidade produtiva, a adaptabilidade a diversos ambientes e, sobretudo, a diversidade de espécies, o que possibilita ao gênero atender a requisitos tecnológicos dos mais variados segmentos da produção industrial madeireira. Esses atributos, de caráter silvicultural, geralmente caminham em direção contrária em relação aos de cunho tecnológico. É comum espécie de elevada capacidade produtiva não ser adequada para dado tipo de uso ou mesmo ter utilidade restrita. Exemplo típico desse tipo inclui o material destinado a celulose e papel e carvão vegetal.

\footnotetext{
${ }^{1}$ Recebido para publicação em 05.11.2003 e aceito para publicação em 10.8.2004.

${ }^{2}$ Departamento de Ciências Florestais da UFLA (trugilho@ufla.br).

${ }^{3}$ Departamento de Engenharia Florestal e Industrial Madeireira da UFPR.

${ }^{4}$ Departamento de Engenharia Florestal da UFPR.
} 
A madeira da grande maioria das espécies de Eucalyptus de rápido crescimento apresenta limitações técnicas quanto à substituição das madeiras tropicais na indústria madeireira. As mais importantes limitações se referem às rachaduras e aos empenamentos (de tábuas e toras), que constituem os principais fatores de redução do rendimento industrial, sendo ambos causados pelas tensões de crescimento.

As tensões de crescimento estão em equilíbrio enquanto a árvore está em pé, mas, tão logo esta é derrubada, ocorrem deformações e rachaduras nos topos de toras, em razão da modificação do estado de equilíbrio que vigorava durante o crescimento (FERRAND, 1983). Assim, a zona periférica da tora, sob tração, tende, após a derrubada, a contrair e a parte central, a expandir, causando as rachaduras de topo nas toras (MALAN, 1979). As tensões de crescimento ocorrem na árvore antes da derrubada, atuando como uma forma de dar-lhes estabilidade (van WYK, 1978). As causas das altas tensões de crescimento não são bem conhecidas, mas há suspeitas de que estejam relacionadas a fatores genéticos, idade, tamanho da tora, taxa de crescimento e inclinação do fuste (OPIE et al., 1984).

Essas tensões de crescimento podem ser determinadas a partir da medição de alterações nos comprimentos de peças de madeira, após a liberação de suas junções a outros elementos vizinhos, dentro do tronco de uma árvore (LISBOA, 1993).

As tensões de crescimento existem e provocam graves entraves na utilização da madeira na forma sólida; isso é de conhecimento geral. O problema está no modo de determinação da magnitude ou níveis dessas tensões. A literatura pertinente informa várias possibilidades para se medirem as tensões de crescimento, apresentando, cada uma delas, dificuldades e facilidades de aplicação. Os métodos de medição sempre se baseiam na determinação indireta das tensões. O método do "Centre de Coopération Internationale em Recherche Agronomique pour le Développemnet, Département dês Forêts - CIRAD-Forêt" ("Growth Strain Gauge" - Medidor de deformação de crescimento), apresenta, como grande vantagem, a facilidade de uso e a rapidez na coleta de dados no campo, pois a avaliação é feita na árvore em pé. Esse método baseia-se na determinação da deformação residual longitudinal (DRL) a uma distância fixa, a qual é diretamente proporcional à tensão de crescimento na direção longitudinal.

Alguns trabalhos têm sido conduzidos, no Brasil, utilizando o método do "CIRAD-Forêt", os quais apresentaram resultados satisfatórios, especialmente, na classificação e seleção de clones de eucalipto, dentre os quais estão os de Souza (2002), Trugilho et al. (2002ab), Lima et al. (2003) e Trugilho et al. (2003). Tais autores tiveram como objetivo comum a avaliação da DRL, em clones, em dada idade e local. Souza (2002) concluiu que o método do "CIRAD-Forêt" se mostrou confiável, de fácil operação e rápido na coleta dos dados de campo.

O objetivo do presente estudo foi verificar os efeitos da idade e da classe diamétrica sobre os níveis das tensões de crescimento longitudinal em árvores de Eucalyptus dunnii oriundas de plantio comercial.

\section{MATERIAL E MÉTODOS}

\subsection{Material experimental e local de amostragem}

O material de estudo foi o Eucalyptus dunnii com $8,13,15$ e 19 anos de idade. Os povoamentos foram implantados por mudas oriundas de sementes, sendo cada árvore considerada um genótipo diferente. Em cada idade foram avaliadas 16 árvores, tendo sido amostradas 64 delas. As árvores foram selecionadas aleatoriamente nos povoamentos, tendo-se o cuidado de escolher as mais representativas de acordo com a classe diamétrica. Não foram consideradas as árvores de bordadura e as que apresentavam sintomas de doenças. Aquelas com 15 anos de idade foram cultivadas na Fazenda São João dos Cavaleiros, enquanto as demais, na Fazenda Rio da Areia.

O material de estudo foi cedido pela empresa Procopiak, localizada no município de Canoinhas, SC, planalto norte catarinense, com latitude de $26^{\circ} 10^{\prime} \mathrm{S}$ e longitude de $50^{\circ} 23^{\prime}$ oeste de Greenwich, a uma latitude de $734 \mathrm{~m}$. O clima, de acordo com a classificação de Köppen, é do tipo Cfa, ou seja, clima temperado com ausência de estação seca, com chuvas bem distribuídas durante o ano inteiro. A temperatura média anual era de $18{ }^{\circ} \mathrm{C}$, e a precipitação pluviométrica variava de 1.800 a $2.400 \mathrm{~mm}$ (SANTA CATARINA, 2003). 


\subsection{Avaliações realizadas nas árvores}

As avaliações foram realizadas a $1,30 \mathrm{~m}$ de altura no tronco das árvores (DAP), sendo medidas nas direções norte, sul, leste e oeste. A avaliação dos níveis das tensões de crescimento foi mensurada indiretamente pela deformação residual longitudinal (DRL), utilizando-se o aparelho "Growth Strain Gauge" (medidor de deformações de crescimento do "CIRAD-Forêt"). Durante as medições, foram medidos os diâmetros (DAP), com fita métrica, de todas as árvores selecionadas.

Na Figura 1, mostram-se os instrumentos utilizados na medição da deformação, em que: 1 - extensômetro; 2 - pinos de fixação (45 mm de distância); 3 - gabarito para fixação dos pinos no tronco das árvores, na direção da grã; e 4 - arco-de-pua com broca de $20 \mathrm{~mm}$.

Os resultados foram avaliados por meio de análise de variância, considerando-se o delineamento inteiramente casualizado com quatro tratamentos (idades) e 16 repetições (árvores-amostra). Ajustaram-se equações de regressão, modelo linear, em que a variável dependente foi a deformação residual longitudinal e a variável independente, a idade e a classe diamétrica.

\section{RESULTADOS E DISCUSSÃO}

\subsection{Avaliação do efeito da idade}

No Quadro 1, apresentam-se os valores médios da deformação residual longitudinal determinada nas

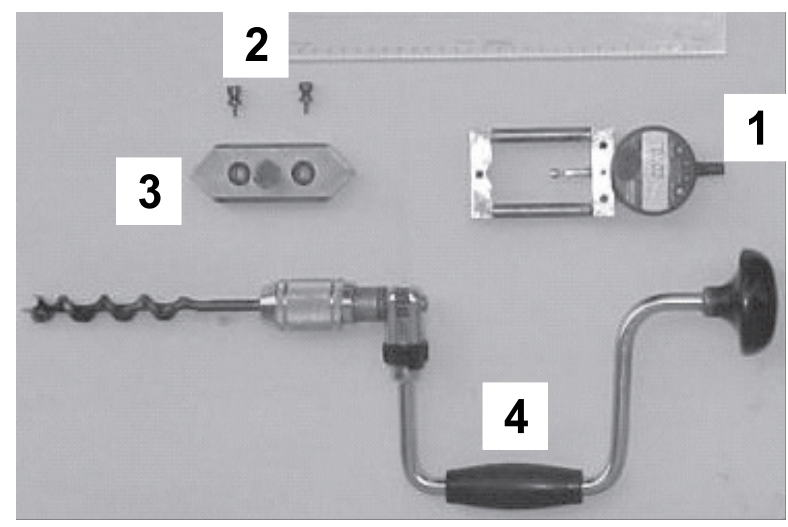

Figura 1 - Instrumentos usados para medição da deformação residual longitudinal (DRL).

Figure 1 - Instruments used for measurement of longitudinal residual strain (DRL). árvores avaliadas e os respectivos coeficientes de variação. Observa-se, nesse quadro, que existe variação entre os materiais avaliados, pois os coeficientes de variação foram elevados com relação à característica em estudo. A DRL média apresentou variação de $31,40 \%$, demonstrando que existe a possibilidade de selecionar as árvores de maior potencial para uso como produtos sólidos, uma vez que a característica está relacionada ao desenvolvimento de rachaduras e empenamentos em peças serradas. A maior variação ocorreu nos 19 anos, com coeficiente de variação $(\mathrm{CV})$ de $35,88 \%$, e a menor nos oito anos $(\mathrm{CV}=23,66 \%)$. A variação entre genótipos ou árvores indica que, dentro das idades pesquisadas, pode-se selecionar material superior para a DRL.

A variação dos valores de CV nos pontos cardinais (norte, sul, leste e oeste) foi praticamente a mesma, variando de 38,75 a $41,32 \%$.

A análise de variância do delineamento experimental adotado (Quadro 2) indicou que o efeito da idade foi não-significativo. Esse resultado é interessante, pois indica a possibilidade de selecionar as árvores precocemente, ou seja, em idades mais novas ou jovens, o que apresenta reflexo no tempo e no custo da etapa do programa de melhoramento florestal.

Apesar da não-existência de diferença estatística significativa da DRL com as idades, foi ajustada uma equação de regressão, modelo linear, para predizer o valor da característica DRL em função da idade.

Quadro 1 - Valores médios e coeficiente de variação (CV) da deformação residual longitudinal $(\mathrm{mm})$ nas árvores em cada idade (anos) avaliada

Table 1 -Average values and variation coefficient $(C V)$ on trees appraised characteristics for each age (year)

\begin{tabular}{cccccc}
\hline Idade & \multicolumn{5}{c}{$\begin{array}{c}\text { Deformação Residual Longitudinal }(\mathrm{mm}) \\
\text { nas Direções Cardinais }\end{array}$} \\
\cline { 2 - 6 } (anos) & Norte & Sul & Leste & Oeste & Média \\
\hline 8 & 0,093 & 0,107 & 0,111 & 0,113 & 0,107 \\
CV (\%) & 38,56 & 26,75 & 35,54 & 25,64 & 23,66 \\
13 & 0,107 & 0,122 & 0,105 & 0,114 & 0,113 \\
CV (\%) & 38,22 & 42,01 & 43,92 & 44,96 & 33,02 \\
15 & 0,115 & 0,108 & 0,112 & 0,108 & 0,111 \\
CV (\%) & 40,43 & 30,66 & 36,22 & 33,46 & 26,64 \\
19 & 0,114 & 0,123 & 0,127 & 0,130 & 0,123 \\
CV (\%) & 41,82 & 44,82 & 43,92 & 43,02 & 35,88 \\
Média & 0,110 & 0,115 & 0,114 & 0,116 & 0,114 \\
CV (\%) & 39,93 & 38,75 & 41,32 & 39,13 & 31,40 \\
\hline
\end{tabular}

R. Árvore, Viçosa-MG, v.28, n.5, p.725-731, 2004 
Quadro 2 - Resumo da análise de variância da idade (anos) Table 2 -Summary of the variance analysis for age (year)

\begin{tabular}{lcc}
\hline Fonte de Variação & Grau de Liberdade & Quadrado Médio \\
\hline Idade & 3 & $0,000758 \mathrm{~ns}$ \\
Resíduo & 60 & 0,001295 \\
Total & 63 & \\
\hline
\end{tabular}

ns = não-significativo.

No Quadro 3, apresenta-se o resumo da análise de variância da regressão. Observa-se também, nesse quadro, que a regressão foi significativa a $24 \%$ de probabilidade e o teste para a falta de ajustamento, não-significativo, indicando que o modelo ajustado é adequado e representativo do fenômeno abordado.

Na Figura 2, mostra-se a influência da idade na DRL, sendo DRLobs e DRLest referentes, respectivamente, à média observada e à média estimada da DRL, pela equação de regressão ajustada (equação 1), a qual apresentou coeficiente de determinação $\left(r^{2}\right)$ de $77,32 \%$.

$$
\hat{\mathrm{Y}}_{\mathrm{i}}=0,0953609+0,00132943 \mathrm{X}_{\mathrm{i}}
$$

em que $\hat{Y}_{i}=$ valor da DRL $(\mathrm{mm})$ e $X_{i}=$ idade da árvore (anos).

Na Figura 2, observa-se que a tendência da DRL é de se elevar com o aumento da idade, indicando que a tensão longitudinal de crescimento tem a mesma tendência.

Ressalta-se que as árvores com DRL média de menor magnitude são as de maior potencial para serem utilizadas como peças serradas. Os valores médios de DRL encontrados em Eucalyptus dunnii foram maiores que o normalmente observado em clones de Eucalyptus

Quadro 3 - Resumo da análise de variância da regressão com o teste para a falta de ajustamento

Table 3 - Summary of the regression analysis of variance with the lack of fit test

\begin{tabular}{lcc}
\hline Fonte de Variação & $\begin{array}{c}\text { Grau de Liberdade } \\
\text { Médio }\end{array}$ & Quadrado \\
\hline Regressão & 1 & $0,001778 *$ \\
Resíduo da regressão & 62 & 0,001266 \\
Falta de ajustamento & 2 & $0,0002603 \mathrm{~ns}$ \\
Resíduo & 60 & 0,001295 \\
Total & 63 & \\
\hline
\end{tabular}

spp. (híbridos naturais) de ocorrência nas Fazendas da Companhia Mineira de Metais do Grupo Votorantim, localizadas no noroeste de Minas Gerais, municípios de Vazante e Paracatu. O valor médio da DRL de um teste clonal composto por 46 clones, dispostos em 105 parcelas, foi de $0,069 \mathrm{~mm}$, com coeficiente de variação experimental de 24,25\% (PÁDUA, 2004). Lima et al. (2003) encontraram valor de 0,071 $\mathrm{mm}$ em cinco materiais genéticos cultivados experimentalmente pela Aracruz Celulose, no Estado do Espírito Santo. Essas diferenças estão relacionadas, principalmente, com a espécie, idade de medição e o local ou sítio.

\subsection{Efeito da classe diamétrica}

No Quadro 4, apresentam-se os valores médios da deformação residual longitudinal (DRL) por classe diamétrica e os respectivos coeficientes de variação. Observa-se, nesse quadro, uma menor variação na idade de oito anos e maior na de 19 anos.

A correlação simples entre o valor da DRL média com o centro da classe e o DAP médio foi de -0,541 e -0,596 para oito anos; -0,879 e -0,908 para 13 anos; 0,318 e 0,321 para 15 anos; e 0,249 e 0,381 para 19 anos de idade. Verificou-se que a correlação foi negativa para 8 e 13 anos e positiva para 15 e 19 anos de idade. Somente nas idades de 8 e 13 anos a correlação simples foi significativa. Nessas idades, a DRL tendeu a diminuir com o aumento do diâmetro, ou seja, árvores de maior incremento diamétrico apresentam menor valor de DRL.

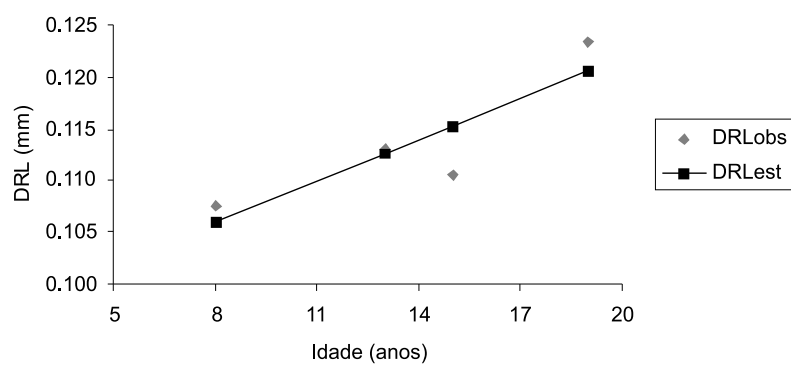

Figura 2 - Valores médios observados (DRLobs) e estimados (DRLest) da deformação residual longitudinal em função da idade (anos).

Figure 2-Observed average values (DRLobs) and estimated (DRLest) of longitudinal residual strain in function of age (year). 
Quadro 4 - Deformação residual longitudinal $(\mathrm{mm})$ por classe diamétrica Table 4 - Longitudinal residual strain $(\mathrm{mm})$ for diametric class

\begin{tabular}{|c|c|c|c|c|c|c|c|c|}
\hline $\begin{array}{c}\text { Idade } \\
(\text { anos })\end{array}$ & $\begin{array}{c}\text { Classe } \\
\text { DAP }\end{array}$ & $\begin{array}{c}\text { Diâmetro } \\
\text { Inferior } \\
(\mathrm{cm})\end{array}$ & $\begin{array}{c}\text { Diâmetro } \\
\text { Superior } \\
(\mathrm{cm})\end{array}$ & Freqüência & $\begin{array}{c}\text { Centro } \\
\text { da Classe } \\
(\mathrm{cm})\end{array}$ & $\begin{array}{l}\text { DAP } \\
\text { Médio } \\
(\mathrm{cm})\end{array}$ & $\begin{array}{l}\text { DRL } \\
\text { Média } \\
(\mathrm{cm})\end{array}$ & $\mathrm{CV}(\%)$ \\
\hline 8 & 1 & 17,50 & 21,60 & 4 & 19,55 & 19,00 & 0,139 & 17,92 \\
\hline 8 & 2 & 21,65 & 25,75 & 3 & 23,70 & 25,00 & 0,096 & 5,09 \\
\hline 8 & 3 & 25,80 & 29,90 & 6 & 27,85 & 27,30 & 0,091 & 22,59 \\
\hline 8 & 4 & 29,95 & 34,05 & 3 & 31,90 & 32,00 & 0,111 & 12,73 \\
\hline 13 & 1 & 29,30 & 34,50 & 4 & 31,90 & 32,10 & 0,120 & 28,61 \\
\hline 13 & 2 & 34,55 & 39,75 & 7 & 37,15 & 36,50 & 0,132 & 31,40 \\
\hline 13 & 3 & 39,80 & 45,00 & 3 & 42,40 & 42,70 & 0,091 & 13,04 \\
\hline 13 & 4 & 45,05 & 50,70 & 2 & 47,65 & 48,30 & 0,069 & 28,59 \\
\hline 15 & 1 & 32,50 & 38,10 & 4 & 35,30 & 34,70 & 0,115 & 38,62 \\
\hline 15 & 2 & 38,15 & 43,75 & 7 & 40,95 & 41,00 & 0,104 & 25,79 \\
\hline 15 & 3 & 43,80 & 49,40 & 2 & 46,60 & 47,00 & 0,096 & 5,16 \\
\hline 15 & 4 & 49,45 & 55,05 & 3 & 52,25 & 53,30 & 0,129 & 23,20 \\
\hline 19 & 1 & 34,10 & 40,70 & 2 & 37,40 & 37,40 & 0,128 & 57,48 \\
\hline 19 & 2 & 40,75 & 47,35 & 7 & 44,05 & 44,40 & 0,124 & 31,02 \\
\hline 19 & 3 & 47,40 & 54,00 & 4 & 50,70 & 48,70 & 0,105 & 57,40 \\
\hline 19 & 4 & 54,05 & 60,65 & 3 & 57,35 & 58,30 & 0,144 & 29,35 \\
\hline
\end{tabular}

No Quadro 5, apresentam-se as equações de regressão ajustadas e o modelo quadrático de cada idade avaliada. Verifica-se, nesse quadro, que nas idades de 8 e 13 anos a regressão foi significativa e os coeficientes de determinação, elevados. Em 15 e 19 anos, a regressão foi não-significativa.

Considerando as regressões significativas, nos oito anos verificou-se que a função passa por ponto de mínimo, no diâmetro de 26,90 cm e DRL de 0,096 $\mathrm{mm}$. Na idade de 13 anos, a função passa por ponto de máximo, no diâmetro de $33,74 \mathrm{~cm}$ e DRL de $0,124 \mathrm{~mm}$.

A Figura 3 ilustra o comportamento da DRL em razão da classe diamétrica de cada idade avaliada. Observa-se, nessa figura, que as funções da DRL média aos 8 (DRL8), 15 (DRL15) e 19 (DRL19) anos apresentam comportamento semelhante, ou seja, as funções passam por ponto de mínimo. Entretanto, nos 13 (DRL13) anos a função passa por ponto de máximo, indicando uma redução da DRL média com o aumento da classe diamétrica. Esse resultado indica que árvores com maiores diâmetros tendem a possuir menores valores da DRL, estando em conformidade com o encontrado por Fernandes et al. (1989). Todavia, Souza (2002) não verificou correlação significativa entre a DRL e as características de crescimento da árvore.

Quadro 5 - Equações de regressão ajustadas das idades (anos) avaliadas

Table 5 - Regression equations adjusted for appraised ages (year)

\begin{tabular}{ccc}
\hline Idade (anos) & Equação A justada & $\mathrm{R}^{2}$ \\
\hline 8 & $\hat{\mathrm{Y}}_{\mathrm{i}}=0,752978-0,0494057 \mathrm{X}_{\mathrm{i}}+0,000918209 \mathrm{X}_{\mathrm{i}}^{2}$ & $99,68^{* *}$ \\
13 & $\hat{\mathrm{Y}}_{\mathrm{i}}=-0,220630+0,0204388 \mathrm{X}_{\mathrm{i}}-0,000302856 \mathrm{X}_{\mathrm{i}}^{2}$ & $89,02^{*}$ \\
15 & $\hat{\mathrm{Y}}_{\mathrm{i}}=0,726719-0,0293783 \mathrm{X}_{\mathrm{i}}+0,000342723 \mathrm{X}_{\mathrm{i}}^{2}$ & $87,38 \mathrm{~ns}$ \\
19 & $\hat{\mathrm{Y}}_{\mathrm{i}}=0,630088-0,0223387 \mathrm{X}_{\mathrm{i}}+0,000240635 \mathrm{X}_{\mathrm{i}}^{2}$ & $66,06 \mathrm{~ns}$ \\
\hline
\end{tabular}

ns, **e * não-significativo e significativos a 1 e $10 \%$ de probabilidade, respectivamente.

$\hat{\mathrm{Y}}_{\mathrm{i}}=$ DRL média da classe diamétrica e $\mathrm{X}_{\mathrm{i}}=$ diâmetro do centro da classe 


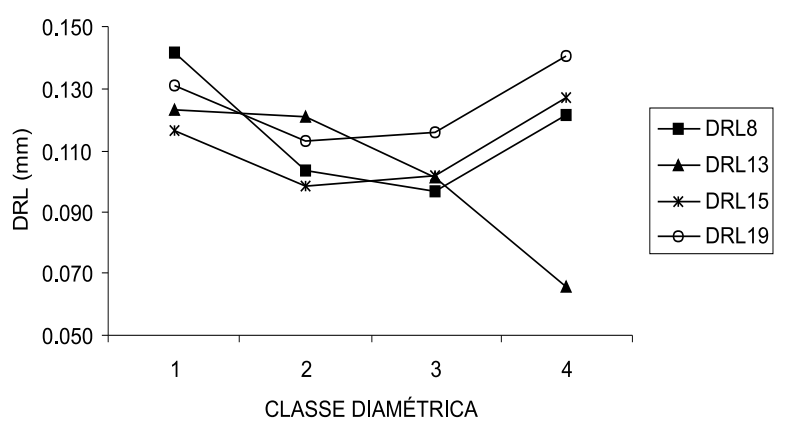

Figura 3 - Comportamento da DRL em função da classe diamétrica. Figure 3-Behavior of DRL in function of diametric class.

\section{CONCLUSÕES}

Existe elevada variabilidade no material estudado, indicando a possibilidade de seleção de material superior para uso como peças serradas.

O efeito da idade foi não-significativo, indicando a possibilidade de seleção de material precocemente.

Existe, na média, uma tendência forte de aumento da DRL em razão da idade.

A correlação simples entre a DRL e o centro da classe e o diâmetro médio da classe foi negativa e significativa nas idades de 8 e 13 anos, respectivamente.

O efeito da classe diamétrica apresentou relação funcional, modelo quadrático, significativa nos 8 e 13 anos e não-significativa aos 15 e 19 anos de idade. Apesar da não-significância da regressão, observouse a existência de uma forte tendência de o modelo quadrático.

\section{AGRADECIMENTO}

Os autores agradem à Empresa Procopiak Compensados e Embalagens S.A. o apoio prestado no desenvolvimento deste trabalho.

\section{REFERÊNCIAS BIBLIOGRÁFICAS}

ASSIS, T. F. Aspecto do melhoramento de Eucalyptus para obtenção de produtos sólidos de madeira. In: TÉCNICAS DE ABATE, PROCESSAMENTO E UTILIZAÇÃO DA MADEIRA DE EUCALIPTO, 1999, Viçosa. Workshop ... Viçosa: DEF/SIF/UFV/IEF, 1999. p. $61-72$.

R. Árvore, Viçosa-MG, v.28, n.5, p.725-731, 2004
FERNANDES, P.S.; FLORSHEIM, S.M.B.; ROCHA, F.T. Tensões de crescimento em procedências de Eucalyptus grandis Hill ex Maiden e suas relações com as características das fibras e densidade básica. Revista do Instituto Florestal, n.1, p.215-234, 1989.

FERRAND, J. C. Growth stresses and silviculture of eucalyptus. Australian Forest Research, v.13, n.1, p.75-81, 1983.

LIMA, J.T. et al. Deformações residuais longitudinais decorrentes de tensões de crescimento em Eucalyptus e suas associações com outras propriedades. Revista Árvore, 2003. No prelo.

LISBOA, C.D.J. Estudo das tensões de crescimento em toras de Eucalyptus grandis Hill ex. Maiden. 1993. 298 f. Tese (Doutorado em Engenharia Florestal) -

Universidade Federal do Paraná, Curitiba, 1993.

MALAN, F.S. The control and-splitting in saw logs: A short literature review. South African Forestry Journal, n.109, p.14 - 8, 1979.

OPIE, J.E.; CURTIN, R.A.; INCOLL, W.D. Stand management. In. HILLIS, W.E.; BROWN, A. G. Eucalypts for wood production. Sydney, CSIRO/Academic Press, 1984. p. 179-197.

PÁDUA, F.A. Estimativas de parâmetros genéticos das tensões de crescimento em clones de Eucalyptus. 2004. 66 f. Dissertação (Mestrado em Tecnologia Ciência e da Madeira) - Universidade Federal de Lavras, Lavras, 2004.

SANTA CATARIANA. Secretaria de Estado da Agricultura e Política Rural. Anuário da agricultura de Santa Catarina, v.1, 287 p. 2003.

SOUZA, M.A.M. Deformação Residual Longitudinal (DRL) causada pelas tensões de crescimento em clones de híbridos de Eucalyptus. 2002, 72 f. Dissertação (Mestrado em Ciência e Tecnologia da Madeira) - Universidade Federal de Lavras, Lavras, 2002. 
TRUGILHO, P.F. et al. Avaliação da tensão de crescimento em clones de Eucalyptus. Floresta e Ambiente, v. 9, n. 1, p. 38-44, 2002a.

TRUGILHO, P.F. et al. Tensões de crescimento: Recentes experiências com medições não destrutivas. In: SÓLIDOS DE EUCALIPTO: AVANÇOS CIENTÍFICOS ETECNOLÓGICOS, 2002, Lavras. Anais ... Lavras: Centro de Estudos em Recursos Naturais Renováveis - CERNE, 2002b. p.125-134.
TRUGILHO, P.F. et al. Relação entre as rachaduras de toras e tábuas com a tensão de crescimento. In: CONGRESSO FLORESTAL BRASILEIRO, 8., 2003, São Paulo. Anais ... São Paulo: Sociedade Brasileira de Engenheiros Florestais e Sociedade Brasileira de Silvicultura, 2003. CD-ROM.

van WYK, J. L. Hardwood sawmilling can have a bright future in South Africa. South African Forestry Journal, n.109, p.47 - 53, 1978. 